Review Article

\title{
Development of Single-Longitudinal-Mode Selection Technology for Solid-State Lasers
}

\author{
Xuanpu Zhang, ${ }^{1,2}$ Zhihan Wang,,2 Shi Liu, ${ }^{1,2}$ Siyu Gou,, ${ }^{1,2}$ Rong Fan,,2 Duo Jin,, \\ Zhenao Bai, ${ }^{3,4}$ and Zhenxu Bai $\mathbb{D}^{1,2,5}$ \\ ${ }^{1}$ Center for Advanced Laser Technology, Hebei University of Technology, Tianjin 300401, China \\ ${ }^{2}$ Hebei Key Laboratory of Advanced Laser Technology and Equipment, Tianjin 300401, China \\ ${ }^{3}$ Aerospace Information Research Institute, Chinese Academy of Sciences, Beijing 100094, China \\ ${ }^{4}$ Beijing GK Laser Technology Co., Ltd., Beijing 102206, China \\ ${ }^{5}$ MQ Photonics Research Centre, Department of Physics and Astronomy, Macquarie University, Sydney, NSW 2109, Australia \\ Correspondence should be addressed to Zhenxu Bai; zxbai@hebut.edu.cn
}

Received 9 October 2020; Revised 22 March 2021; Accepted 8 April 2021; Published 28 July 2021

Academic Editor: Jayanta K. Sahu

Copyright ( $) 2021$ Xuanpu Zhang et al. This is an open access article distributed under the Creative Commons Attribution License, which permits unrestricted use, distribution, and reproduction in any medium, provided the original work is properly cited.

\begin{abstract}
Lasers with narrow linewidths and single frequencies are widely used in fields such as radar detection, nonlinear optics, and precision measurements. The demand for such lasers has promoted the rapid development of single-longitudinal-mode (SLM) selection technology. Here, we highlight the working principles of current mainstream SLM selection technologies and the recent advances in the field. We compare the characteristics of different SLM selection methods and list the challenges faced by these technologies.
\end{abstract}

\section{Introduction}

With only one fundamental frequency component, singlelongitudinal-mode (SLM) lasers have the advantages of narrow linewidths and low phase noise and are therefore ideal for applications where a high-coherence light source is required, including Doppler wind lidar, atmospheric composition measurement, coherent optical communication, gravitational wave detection, sodium guide star, and nonlinear optics [1-5]. In general, if the linewidth of an SLM laser is narrower than the resolution of a spectrum analyzer or the nonlinear gain bandwidth of a specific medium, it is assumed to be a single-frequency laser. The generation of SLM lasers is usually based on a combination of the linewidth of the gain medium and the losses of the cavity. Alternatively, SLM selection methods can be applied to solid state lasers to generate SLM lasers [6, 7]. Owing to their strong power (average and peak) scaling ability and wide range of wavelength coverage, solid-state lasers play an important role in space exploration, defense, and manufacturing [8-10]. In the past few decades, there has been a rise in interest towards the SLM selection of solidstate lasers, which also promotes the development of optical components, cavity structures, and active controllers.

In a standing wave cavity, the frequency interval between each longitudinal mode (oscillation frequency) is described by

$$
\Delta v=\frac{c}{2 n L},
$$

where $c$ is the speed of light in vacuum, $n$ is the overall refractive index of the cavity, and $L$ is the cavity length, as shown in Figure 1(a). Usually, the resonator contains a series of discrete longitudinal modes within the range of the gain curve above the threshold. That is, in the case of a freerunning laser (without SLM selection), a series of longitudinal modes with an interval of $\Delta v$ could oscillate in the spontaneous emission spectrum of the gain medium, as shown in Figure 1(b). In addition, spatial hole burning (SHB) is also a cause of multiple longitudinal modes in the cavity. The SHB effect, which results from the stronger gain saturation at locations with higher laser intensities, leads to a 


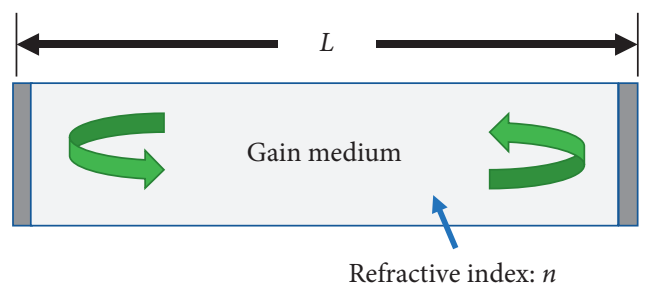

(a)

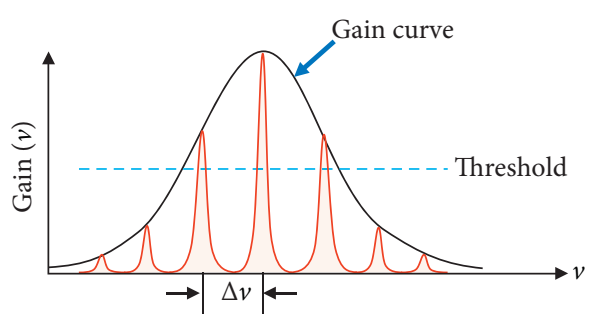

(b)

FIgURE 1: Schematic of (a) oscillator structure and (b) oscillation spectrum.

spatial pattern of excitation density [11]. Owing to the SHB, different longitudinal modes can use activated particles in different spaces to generate oscillations at the same time.

At present, controlling the threshold and introducing mode competition are the main approaches to generate SLM laser output. Therefore, special cavity design or the introduction of mode selection components has to be carried out to generate SLM lasers. The common approaches to realize SLM operation for solid-state lasers include short cavity, intracavity etalon, unidirectional ring cavity, twisted-mode cavity, and seed injection [12-18]. Although many technical methods have been reported, no paper has yet provided an overview of the various SLM selection techniques and characteristics (viz. precision, cost, output power, ease of fabrication, etc.) of different methods. This paper summarizes the SLM selection techniques and reviews the developments of some typical SLM lasers, including the future prospects of the lasers in various applications.

\section{SLM Selection Technologies}

2.1. Short Cavity Method. According to equation (1), the longitudinal mode interval $\Delta v$ is inversely proportional to the optical length $n L$ of the resonator. Therefore, by shortening the cavity length, the frequency interval between adjacent longitudinal modes can be made larger than that of the gain linewidth of the medium. As shown in Figure 2, only one longitudinal mode in the gain curve reaches the oscillation threshold. This SLM selection method is called the short cavity method. To realize a large enough longitudinal mode interval via the short cavity method, a millimeter-scale cavity length is usually required for a solid-state laser.

In 1979, Kubodera and Otsuka [19] proposed a laser diode (LD) end-pumped microchip $\mathrm{LiNdP}_{4} \mathrm{O}_{12}$ SLM laser with a cavity length of only $300 \mu \mathrm{m}$. This experimental setup is shown in Figure 3. Stable continuous-wave (CW) SLM outputs of $2 \mathrm{~mW}(1.048 \mu \mathrm{m})$ and $0.5 \mathrm{~mW}(1.317 \mu \mathrm{m})$ were obtained. In 2010, Li et al. [20] proposed a Tm,Ho: $\mathrm{YVO}_{4}$ microchip laser with a cavity length of $0.5 \mathrm{~mm}$. In their study, a $1.2 \mathrm{~W}$ multilongitudinal mode output was obtained at a cryogenic temperature of $77 \mathrm{~K}$. The laser produced a single-frequency $8 \mathrm{~mW}$ output at $2052.6 \mathrm{~nm}$ when the temperature was increased to room temperature $\left(15^{\circ} \mathrm{C}\right)$. In 2010, Wang et al. [21] proposed a SLM lasing of CW Tm,Ho: YAP microchip laser with emission at $2000.4 \mathrm{~nm}$. In 2015, You et al. [22] developed an LD end-pumped SLM microchip laser with emission at $2.7 \mu \mathrm{m}$ using a $600 \mu \mathrm{m}$ thick

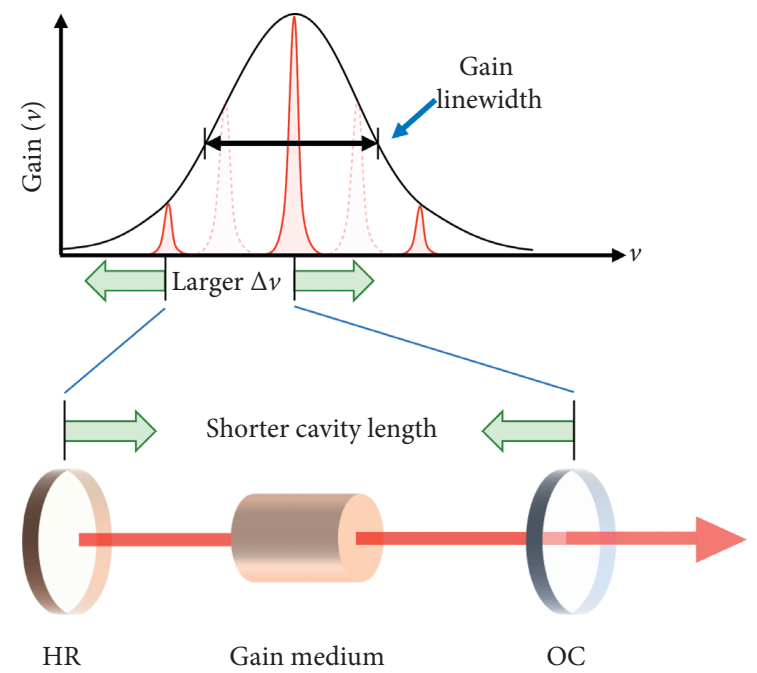

FIGURE 2: Schematic showing the principle of SLM selection by short cavity method.

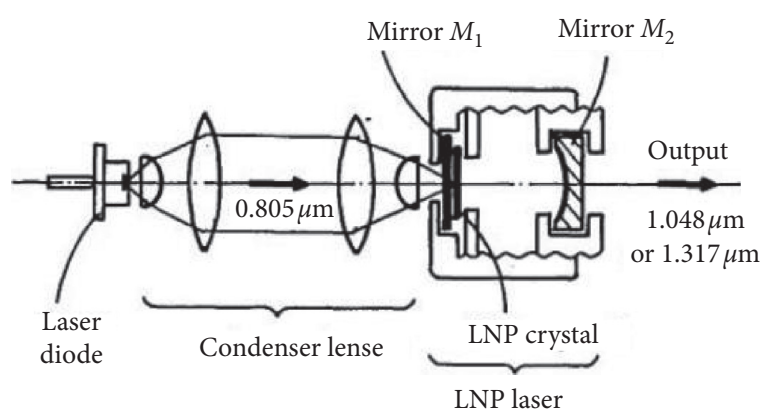

Figure 3: Structure of LD-pumped $\mathrm{LiNdP}_{4} \mathrm{O}_{12}$ lasers for SLM operation [19].

heavily doped Er:GGG crystal. A maximum output power of $50.8 \mathrm{~mW}$ and a maximum pulsed energy of $0.306 \mathrm{~mJ}$ were demonstrated with pulse repetition rates of 300,200 , and $100 \mathrm{~Hz}$, respectively. In 2019, Chen et al. [23] realized a $1521 \mathrm{~nm}$ SLM microchip Q-switched lasing by a $\mathrm{Co}^{2+}$ : $\mathrm{MgAl}_{2} \mathrm{O}_{4}$ saturable absorber in a $1.52 \mathrm{~mm}$ thick Er:Yb: $\mathrm{YAl}_{3}\left(\mathrm{BO}_{3}\right)_{4}$ crystal. A maximum single pulse energy of $16.5 \mu \mathrm{J}$ at a repetition rate of $26.3 \mathrm{kHz}$ and pulse duration of 2.9 ns was generated.

As there is no need to insert any mode selector in the cavity, SLM solid-state lasers based on short cavities are developing rapidly since the invention of the laser. However, 
the reduced geometric cavity length and gain medium size of such microchip structures lead to low output power $(\sim \mathrm{mW})$. Meanwhile, the limited size makes it difficult to add nonlinear optical elements or modulation devices into the cavity to adjust the output characteristics of the output beam.

2.2. Intracavity Etalon Method. Fabry-Perot (FP) etalon, based on the interference mode effect, can be used as the longitudinal mode selection element [24-26]. When an FP etalon is inserted, only the longitudinal mode with the highest transmittance oscillates in the cavity, while the other modes cannot reach the threshold because of the extremely low transmittance. That is, the FP etalon increases the net gain difference between different longitudinal modes (via free spectral range (FSR)) to achieve the SLM output. The principle of FP etalon mode selection is shown in Figure 4.

As early as 1963, Collins and White [27] used a FP etalon as the mode selector, which was composed of $28.6 \mathrm{~mm} \times 3.2 \mathrm{~mm}$ quartz flats with reflectivity of 70,85 , and $93 \%$. They successfully realized single-frequency output while reducing the beam divergence angle at the diffraction limit. In 1970, Danielmeyer [28] proposed a theoretical model for longitudinal mode selection and frequency stabilization based on the intracavity etalon design and experimentally demonstrated linearly polarized singlefrequency output power of $150 \mathrm{~mW}$ near the center of the gain curve. In 2009, Yao et al. [29] developed a narrow linewidth CW Tm:YLF laser with double FP etalons (thicknesses of $0.5 \mathrm{~mm}$ and $0.1 \mathrm{~mm}$ ). The laser operated at $1.9 \mu \mathrm{m}$ with full width at half maximum (FWHM) of approximately $0.15 \mathrm{~nm}$ and maximum output power of $14.0 \mathrm{~W}$. At present, SLM outputs via the intracavity etalon method have been demonstrated with different gain media such as Tm, Ho:YLF [30], Er:YAG [31], and Ho:LuAG [32].

The etalon-based SLM laser has the characteristics of a simple overall structure, high compactness, and customized wavelength (for example, the optical material, thickness, and reflectivity of the etalon can be designed according to the cavity property). However, due to the additional intracavity loss induced by the etalon and limited threshold gap between adjacent longitudinal modes, it is difficult to generate highpower SLM output directly. Therefore, this technique is often accompanied by power amplifiers when higher power is required for specific applications.

\subsection{Traveling Wave Cavity. In a homogeneously broadened} laser, the mode competition caused by the gain saturation effect helps to produce SLM oscillation. However, because of the SHB effect caused by the standing wave, the laser inevitably generates multiple-longitudinal modes in the output when the pump power is sufficiently high. To eliminate the SHB effect, the beam can be designed to propagate along a single direction in the resonator, forming a traveling wave resonant cavity. Based on this idea, different structures have been developed for SLM generation including a nonplanar

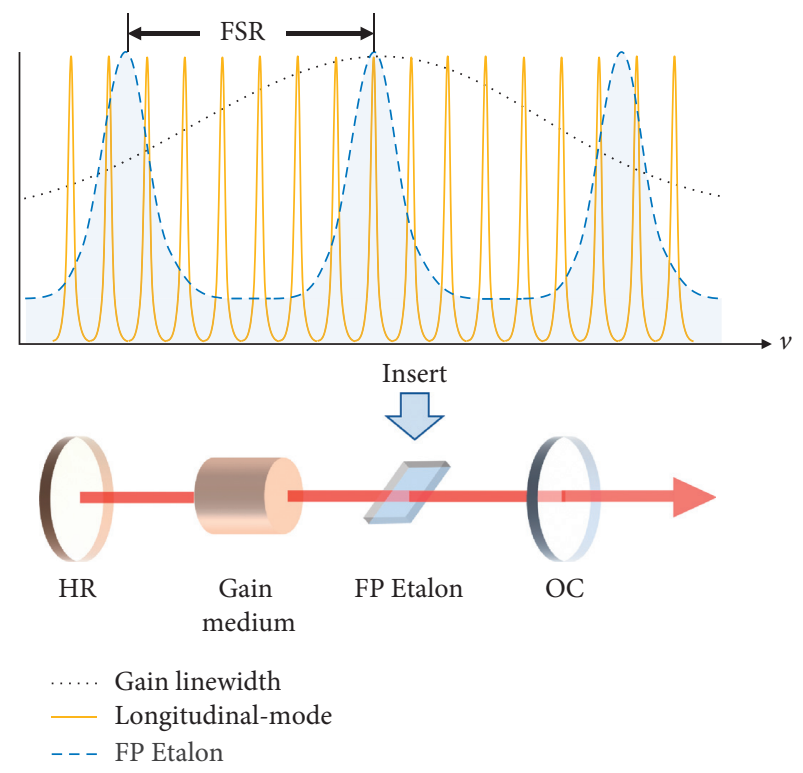

FIgURe 4: Schematic of SLM selection by Fabry-Perot etalon.

ring oscillator (NPRO), discrete ring cavity, and twisted mode cavity.

2.3.1. NPRO. NPRO is consisting of a single laser crystal within which the laser circulates [17, 33]. The front face of the laser crystal has a dielectric coating (see Figure 5), which serves as the output coupler and also as a partially polarizing element, facilitating unidirectional oscillation. In addition, all the other internal surfaces facilitate total reflection. With the action of an external magnetic field, unidirectional operation along one single polarization can be obtained easily by suppressing other polarizations, thus avoiding any standing-wave patterns that cause SHB.

In 1985, Kane and Byer [17] took the lead in realizing the LD-pumped Nd:YAG monolithic nonplanar ring cavity laser. A single-frequency laser output of $163 \mathrm{~mW}$ at $1064 \mathrm{~nm}$ was obtained. Then, they improved the frequency stability by using a similar cavity type and structure, generating a singlefrequency-stabilized laser with a frequency fluctuation of $40 \mathrm{kHz}$ [34]. In 2013, Wang et al. [35] demonstrated an $8.0 \mathrm{~W}$ single-frequency laser operating at $2.1 \mu \mathrm{m}$ from an Ho: YAG-based NPRO. The experimental setup is shown in Figure 6. In the past two decades, different crystals have also been applied in NPRO lasers for single-frequency generation, including Tm:YAG $(2.0 \mu \mathrm{m}$ emission) [36], Er:YAG (1.6 $\mu \mathrm{m}$ emission) [37], and Nd:YAG $(1.1 \mu \mathrm{m}$ and $1.3 \mu \mathrm{m}$ emissions) [38, 39].

NPRO lasers are highly compact with high stability offering low loss which can be used to generate high-power SLM output. In addition, slow and fast tuning of the laser frequency can be realized by controlling the crystal temperature and piezoelectric ceramic of NPRO lasers. Therefore, SLM lasers based on NPRO show good application prospects in the field of coherent optical communication. 


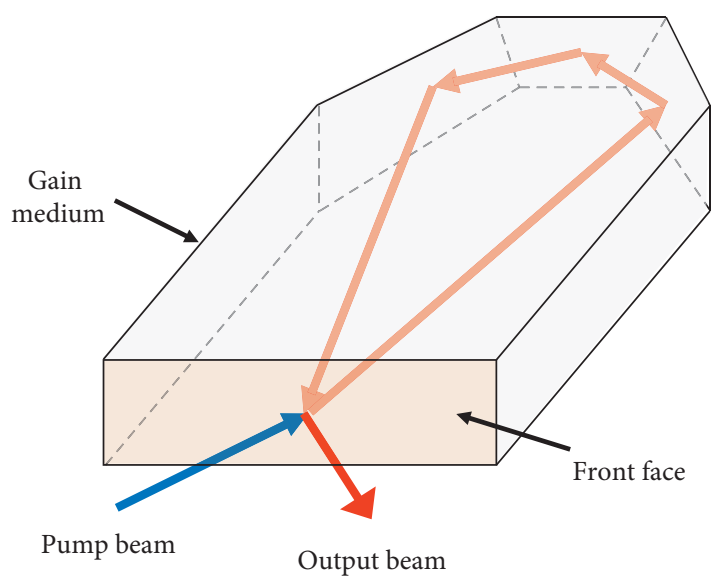

Figure 5: Schematic of an NPRO

2.3.2. Discrete Ring Cavity (Unidirectional Operation). In recent years, ring resonance cavities based on discrete components have been developed for generating SLM output as well. Unidirectional operation is most commonly implemented through the use of an intracavity Faraday rotator. In 1972, Clobes and Brienza [40] achieved single-frequency operation of a Brewster-cut Nd: YAG laser by using a ring cavity configuration containing a small differential loss based on the use of a Faraday rotator. In 2009, Zhao et al. [41] used a four-mirror ring cavity generating $13.6 \mathrm{~W}$ single-frequency output with $\mathrm{Nd}: \mathrm{YVO}_{4} / \mathrm{YVO}_{4}$ bonded crystals as the gain medium. In the same year, Shardlow and Damzen [42] reported a single-frequency output of $17 \mathrm{~W}$ by constructing a threemirror ring cavity in Nd: $\mathrm{YVO}_{4}$ slat crystals. In 2010, Zhao et al. [43] developed a $12 \mathrm{~W}$ single-frequency $1064 \mathrm{~nm}$ laser by using a four-mirror ring cavity with a conversion efficiency up to $52.7 \%$.

Different from NPROs, this free-space traveling wave cavity structure is also conducive for the insertion of nonlinear crystals to achieve efficient intracavity nonlinear frequency conversion, thereby extending the wavelength range of SLM lasers. In addition, the frequency doubling process performed in the traveling wave cavity helps the fundamental frequency light to achieve stable single-frequency operation. The introduced nonlinear losses can suppress the inactive longitudinal mode of the fundamental frequency light in the cavity, thereby reducing the possibility of multilongitudinal mode oscillation or mode jumping [44]. In 2011, Liu et al. [45] demonstrated a CW single-frequency $532 \mathrm{~nm}$ laser with a six-mirror ring resonator and an intracavity frequency doubler. In their study, an etalon was inserted into the cavity to narrow the gain spectra and suppress mode hopping. The experimental setup is shown in Figure 7. In 2013, Wang et al. [46] developed a $25.3 \mathrm{~W}$ intracavity frequency doubling singlefrequency green laser in a four-mirror ring cavity, combined with controlling the boundary temperature.

SLM lasers based on unidirectional operation discrete ring cavities have the advantages of flexible cavity design and pump structure, which is beneficial for achieving higher output power over a wide wavelength range.
2.3.3. Twisted Mode Cavity. The twisted mode cavity is another common method used to eliminate the SHB effect. The SLM laser operation is realized by inserting a polarizer into the cavity, and two quarter-wave plates at the two ends of the gain medium, respectively. In this way, the light field in the gain medium is no longer in the standing wave mode, thus eliminating the SHB effect. A schematic of the twisted mode cavity setup is shown in Figure 8.

In $2005, \mathrm{Wu}$ et al. [47] demonstrated a $2.1 \mathrm{~W}$ single frequency $1.06 \mu \mathrm{m}$ laser based on the twisted mode cavity. In 2011, Gao et al. [48] reported a twisted mode SLM laser at $2 \mu \mathrm{m}$ with Tm:YAG as the gain medium. A $1.46 \mathrm{~W}$ singlefrequency laser was obtainedwith $19.2 \%$ slope efficiency. In 2019, Luo et al. [49] designed anLD-pumped SLM Pr:YLF laser that directly oscillates at $640 \mathrm{~nm}$. The diagram is shown in Figure 9. Under $3.5 \mathrm{~W}$ blue pumping, $403 \mathrm{~mW}$ SLM operation with a linewidth of $150 \mathrm{MHz}$ and a slope efficiency of $26.8 \%$ was obtained.

SLM laser based on twisted mode cavity is sensitive to the intracavity polarization state, so it is greatly affected by the thermally-induced birefringence of the gain medium, which limits the power scaling.

2.4. Volume Bragg Grating (VBG) Method. VBG with high diffraction efficiency is an alternative solution to realize SLM lasing. Any wavelength change that violates the Bragg condition will result in a significant drop in diffraction efficiency. Therefore, the VBG can be used as a "filter" to achieve high reflection at a specific wavelength or band and shows good spectral and angular selectivity. Both reflective and transmissive VBGs can be used for mode selection. The working principle of the VBG is shown in Figure 10.

In 2013, Sun et al. [50] useda reflective volume Bragg grating (RBG) as the output mirror and used active Q-switching to obtain a stable $1063.9 \mathrm{~nm}$ SLM pulse output. The experimental setup is shown in Figure 11, and the optical cavity length of the device was $38 \mathrm{~mm}$. The repetition frequency after Q-switching was $5-150 \mathrm{~Hz}$, the peak power was $0.66 \mathrm{MW}$, and the pulse width was $645 \mathrm{ps}$. When the optical length reaches $100 \mathrm{~mm}$, multiple modes will be generated. The gain crystal length is only $2.6 \mathrm{~mm}$, which limits the further increase in power. In 2015, Jin et al. [51] used RBG and graphene passive Q-switching to obtain a single-frequency laser pulse with an output power of $724 \mathrm{~mW}$, single pulse energy of $7.5 \mu \mathrm{J}$, repetition frequency of $96.2 \mathrm{kHz}$, and duration of $2.2 \mu \mathrm{s}$. Passive Q-switching increases the pulse set-up time, which is conducive to the realization of a SLM. However, the passive Q-switched output pulse has a time jitter, which limits its application range.

Owing to the limitation of the gain medium of the laser, the length of the resonant cavity cannot be further shortened. This may cause the grating bandwidth to be longer than the longitudinal mode interval. It is impossible to achieve SLM operation with a single VBG. Therefore, multiple VBG are required, and mode selection is carried out by a combination of these gratings. In 2009, Hui et al. [52] proposed a SLM laser based on a combined VBG, and its 


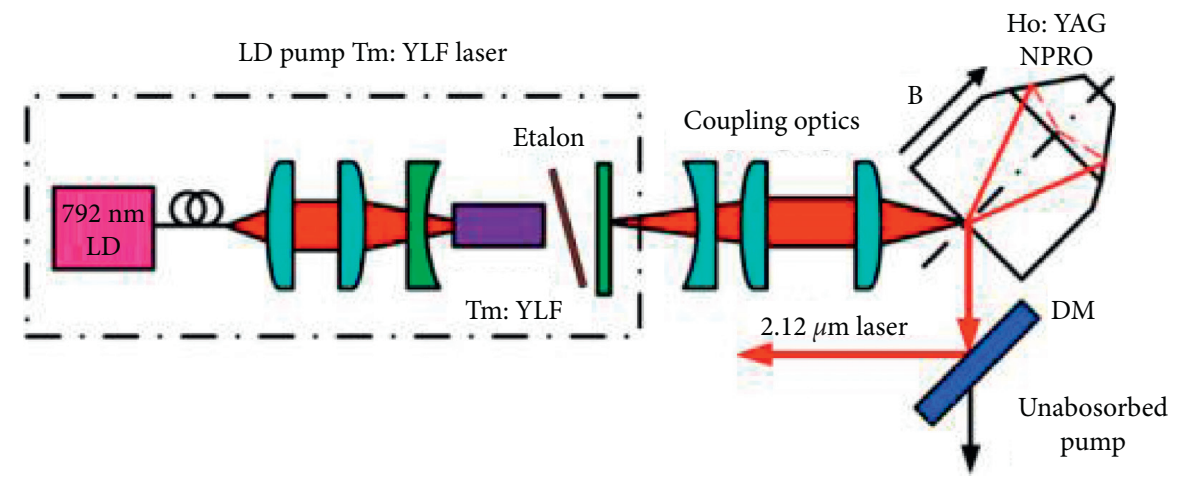

FIgURE 6: The experimental setup of a Ho:YAG NPRO laser [35].

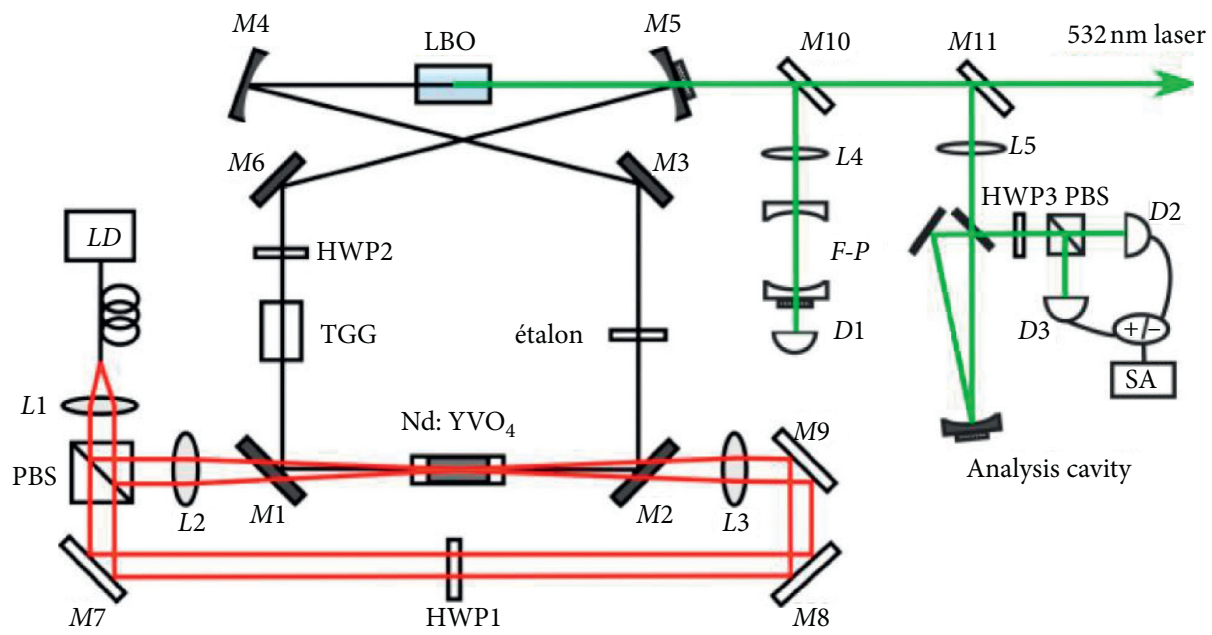

FIGURE 7: Schematic of the structure of a six-mirror ring cavity single-frequency green laser [45].

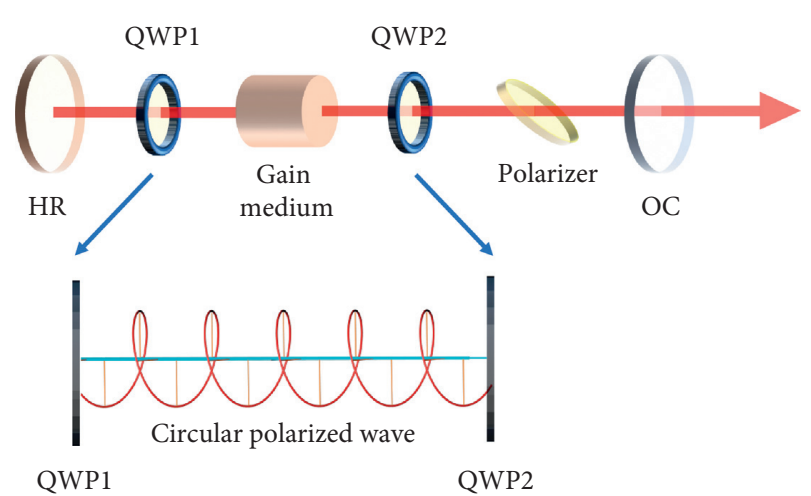

FIGURE 8: Schematic showing the principle of the twisted mode cavity.

structure is shown in Figure 12. The combination of transmission and reflection gratings is used to select the output light angle and wavelength, so that the laser realizes SLM oscillation and obtains an output with a wavelength of $1053 \mathrm{~nm}$ and a pulse energy of $2 \mathrm{~mJ}$.

The Bragg grating is limited by the accuracy of the mode selection and is generally used as a part of the composite mode selection structure. The RBG is usually used as the output mirror of the resonant cavity.
2.5. Seed Injection and Amplification Method. The output powers of the abovementioned SLM selection techniques in a single cavity are relatively low. To generate higher power output, the seed injection and amplification method is proposed. The basic principle is as follows. A single-frequency low-power seed with excellent temporal and spatial characteristics is injected into the resonator. The cavity length continuously changes near the descending edge of the pumping pulse and detects the match of the seed light and the oscillation mode in the cavity. In this case, when the seed light resonates in the cavity, the $Q$ switch is turned on, thereby outputting a single-frequency pulse laser with a linewidth near the Fourier transform limit. Park et al. [53] showed that the frequency of the SLM output by the seed injection solidstate laser is not exactly the same as the seed light frequency, but is the longitudinal mode frequency closest to the seed light frequency. Seed injection technology plays a vital role in the energy stability and frequency stability of the output laser and is the core technology of singlefrequency lasers. Commonly used seed injection techniques include minimum setup time, resonance detection technology, and optimized resonance detection technology. A schematic of this technology is shown in Figure 13. 


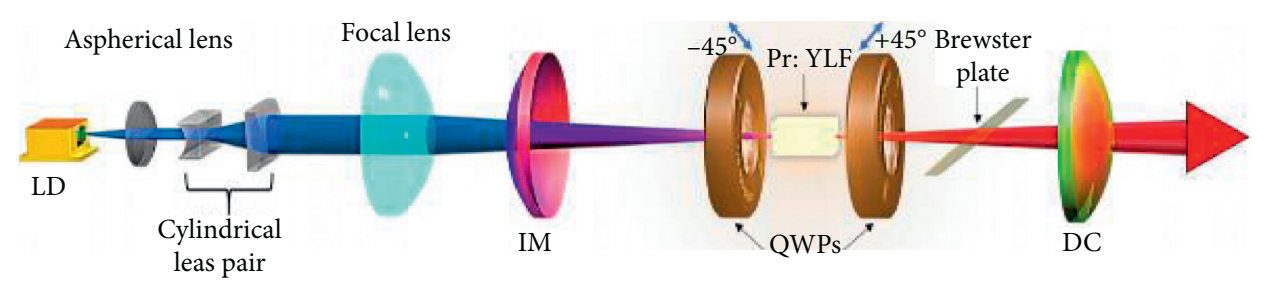

Figure 9: Schematic of SLM laser based on torsion pendulum cavity [49].

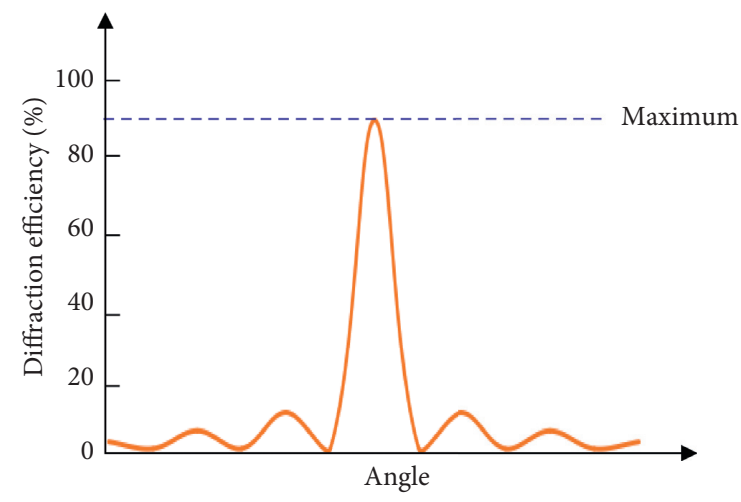

(a)

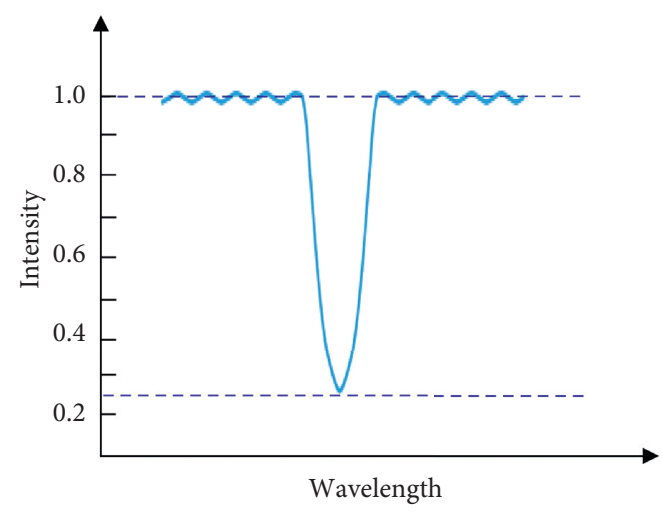

(b)

FIgURE 10: Schematic of reflecting VBGs with (a) angular selectivity and (b) spectral selectivity.

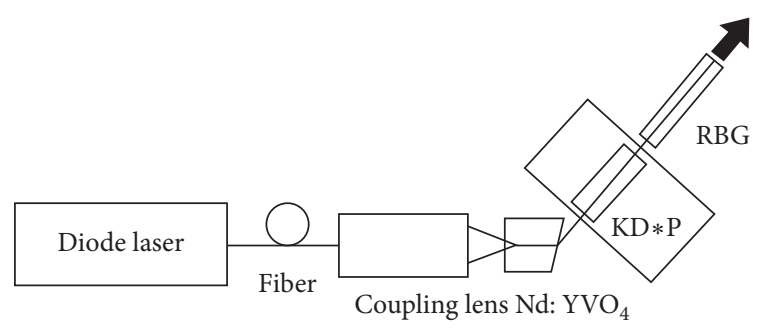

FIGURE 11: SLM laser based on RBG [43].

In 2007, Schröder et al. [54] designed a seed-injected Nd: YAG single-frequency ultraviolet laser for airborne Doppler wind lidar using the technology of minimizing the settling time. A $100 \mathrm{~Hz}$ pulse with a duration of $35 \mathrm{~ns}$ and a line width measured by beat frequency to be less than $15 \mathrm{MHz}$ was passed through a two-stage dual-pass amplifier and nonlinear crystal. This resulted in an output of $355 \mathrm{~nm}$ ultraviolet laser with pulse energy of $60 \mathrm{~mJ}$ and pulse width reduced to $25 \mathrm{~ns}$.

In 1986, Henderson et al. [55] proposed a scan-triggered resonance detection technology. In 2017, Gao's research group developed a seed-injected Ho:YAG single-frequency laser [56], using a fiber with a line width of approximately $10 \mathrm{kHz}$. The coupled output Ho:YAG monolithic nonplanar ring cavity (NPRO) laser is used as the seed source, and the driven cavity is an acousto-optic Q-switched butterfly fourmirror ring cavity pumped by a continuous-wave Tm:YLF laser. The structure is shown in Figure 14. Ramp-fire resonance detection technology was used to achieve a stable $2.09 \mu \mathrm{m}$ SLM laser output with output pulse energy of $6.24 \mathrm{~mJ}$, pulse width of $172 \mathrm{~ns}$, and repetition frequency of $1 \mathrm{kHz}$. The spectral linewidth was measured as $2.61 \mathrm{MHz}$ using the optical beat frequency method, and the energy stability was less than $3 \%$. The energy stability index has room for improvement.

To solve the problem of output laser energy jitter caused by the unstable $Q$-switched trigger signal of the Ramp-fire resonance detection technology, researchers have proposed several improvements. In 1997, Fry et al. [57] proposed a scan-hold-fire (Ramp-hold-fire) resonance detection technology based on Ramp-fire resonance detection technology. The principle of this technology is similar to Ramp-fire resonance detection technology. The difference is that when the interference peak is detected, the $Q$ switch is not turned on immediately, but the scanning voltage applied to the piezoelectric ceramic is maintained at the same level as that at the time when the interference peak is detected. At this time, the longitudinal mode frequency of the driven cavity remains consistent with the seed light frequency until the $Q$ switch trigger signal arrives. The technical working principle is shown in Figure 15.

In 2016, Zhang et al. [59] developed the first seed-injected Ho:YAG ceramic laser using this technology. The laser used a $2.09 \mu \mathrm{m}$ seed laser with an output pulse energy of $14.76 \mathrm{~mJ}$, pulse width of $121.6 \mathrm{~ns}$, and repetition frequency of $200 \mathrm{~Hz}$. The linewidth was $3.84 \mathrm{MHz}$ for one-hour operation.

In order to further improve the stability of the output laser energy and avoid the influence of external interference as much as possible, Ertel et al. [60] proposed a resonant detection technology with feedback in 2005. In 2007, Zhou et al. [61] proposed a delay-sweep-trigger (delay-ramp-fire) resonance detection technology and an improved scheme of this technology, the double piezoelectric ceramics (PZT) resonance detection technology with bias feedback [62]. In 2016, 


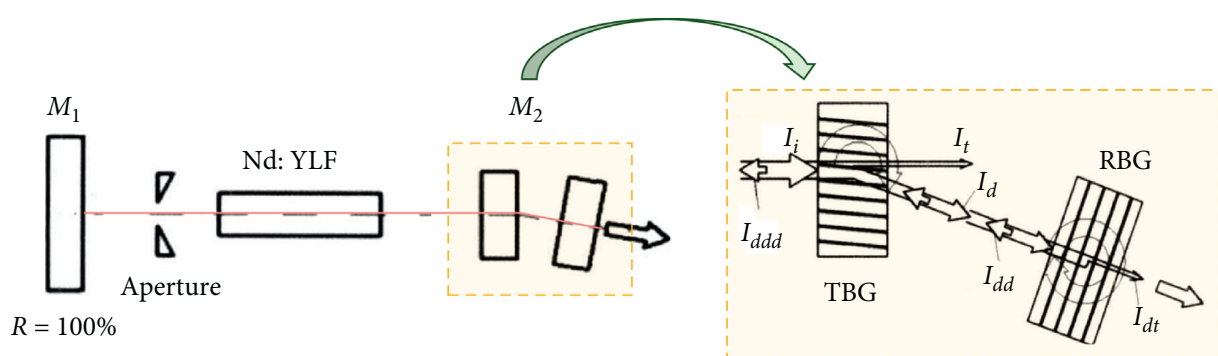

Figure 12: SLM laser based on combined VBGs [52].

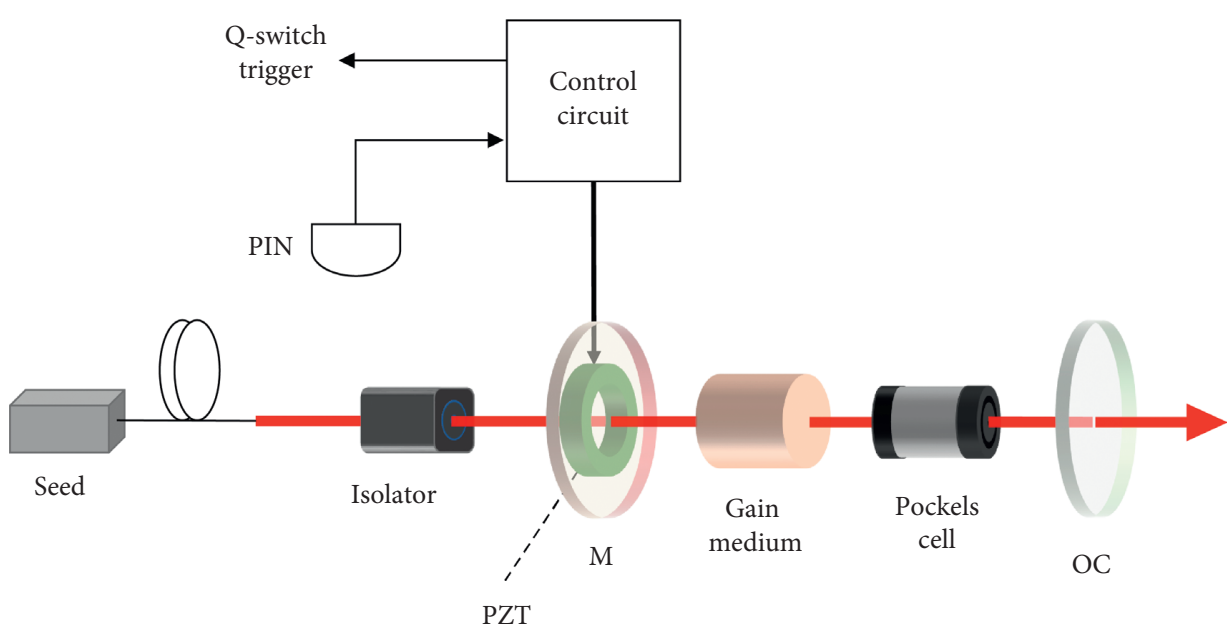

FIGURE 13: Schematic of seed amplification technology.

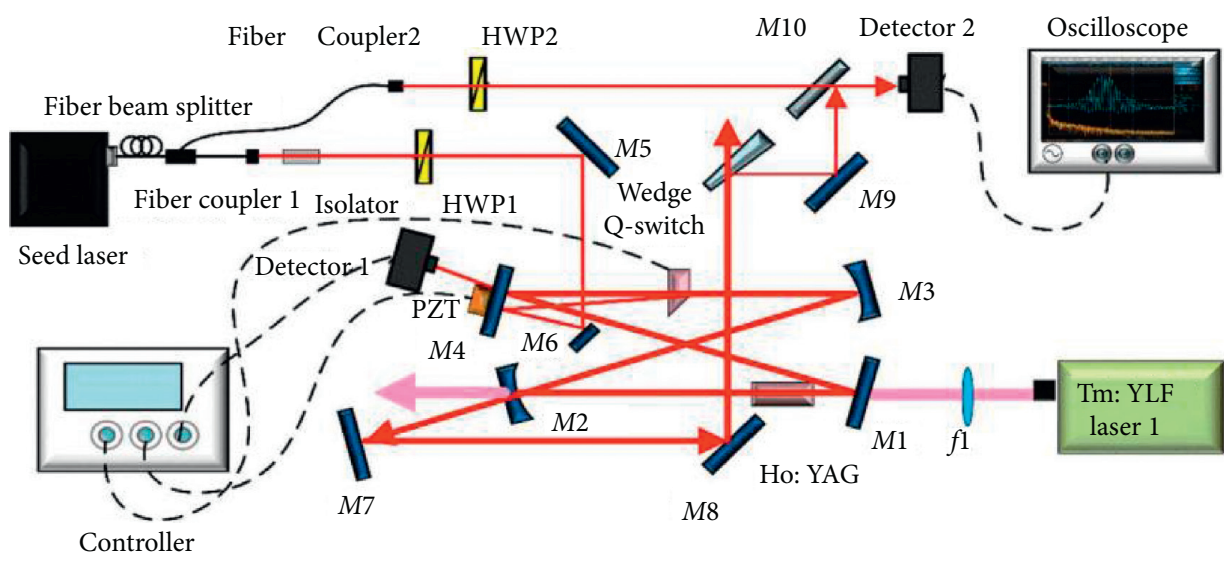

FIGURE 14: Ho:YAG single-frequency laser with seed injection [56].

Gao et al. [63] combined the real-time resonance tracking detection method and proposed a new seed injection technology: a double PZT sinusoidal scanning resonance detection technology with bias voltage feedback. The singlefrequency laser developed by this technology has an output pulse energy of $0.7 \mathrm{~mJ}$, a pulse width of $27 \mathrm{~ns}$, a repetition frequency of $400 \mathrm{~Hz}$, and a line width of $18 \mathrm{MHz}$. A frequency jitter of less than $9.1 \mathrm{MHz}$ was measured within 30 mins. In 2014, Gibert et al. [64] used Pound-Drever-Hall (PDH) injection locking technology to develop a dual-wavelength single-frequency Ho:YLF laser. The standard deviation of the output laser frequency stability was $2 \mathrm{MHz}$, and the frequency jitter range within $10 \mathrm{~s}$ was less than $70 \mathrm{kHz}$, which is very suitable for differential absorption radar to detect atmospheric $\mathrm{CO}_{2}$ concentration. In 2017, in the German Aerospace Center Lemmerz et al. [65] adopted the scan-delaytrigger (Ramp-delay-fire, $\mathrm{RDF}$ ) resonance detection technology to develop an all-solid-state ultraviolet laser for airborne wind measurement radar. The oscillation amplification structure, combined with type I LBO frequency double 


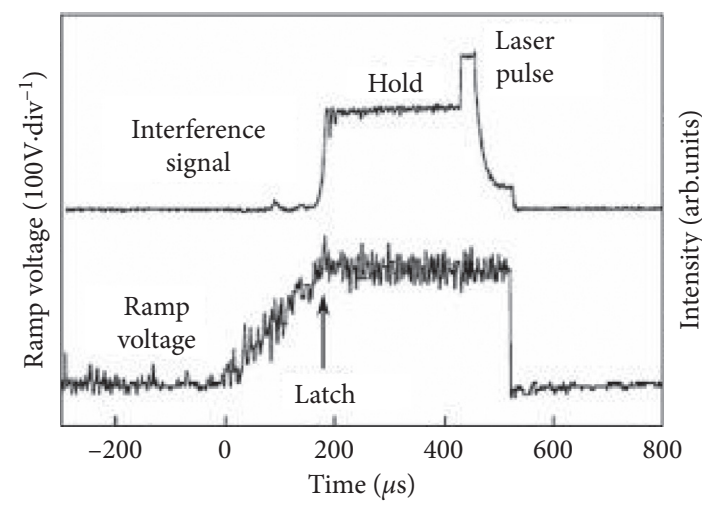

FIGURE 15: Schematic showing the principle of the Ramp-hold-fire resonance detection technology [58].

crystal and type II LBO sum frequency crystal, achieved an ultraviolet laser with an output pulse energy of $60 \mathrm{~mJ}$, pulse duration of $20 \mathrm{~ns}$, and repetition frequency of $355 \mathrm{~nm}$ at $50 \mathrm{~Hz}$.

Figure 16 shows the principle of the trigger mechanism of the $\mathrm{RDF}$ resonance detection technology. $T_{1}$ is the pump start time, $T_{2}$ is the PZT sweep voltage start time, peak detection starts at a fixed time $T_{3}$, and the first peak is detected at $T_{4}$. In order to trigger the radar return light detector at a precise time before the pulse is generated, the trigger time $T_{6}$ of the next pulse is predicted based on the difference between $T_{3}$ and $T_{4}$ to determine the time $T_{5}$ to trigger the return light detector, and the cavity is changed within the delay time $D_{2}$ to compensate for the change in the cavity length caused by the PZT during this period (including the pulse setup time). This method can reduce the pulse time jitter caused by the external interference in the ramp-fire technology and help in finally realizing a single-frequency laser emission source with stable frequency and time.

The seed injection method can effectively increase the output power of the laser, and the beam quality of the seed laser has a great influence on it. Therefore, it is very important to use a suitable seed laser injection technology. In current technologies, the seed injection method is often cascaded with nonlinear crystals to form a frequencydoubled high-power laser [66].

2.6. Compound Cavity Method. Compared to a resonant cavity with only one light propagation path, the compound cavity has two or more optical transmission paths that have common parts. Different optical transmission resonators are called subcavities, and a compound cavity consists of multiple subcavities. Depending on whether the light propagation path is separated in the direction, the compound cavity can be divided into the same direction as the compound cavity and the bifurcation composite cavity. A typical compound cavity mode selection structure is shown in Figure 17. The mirror at one end of the laser is replaced by a combination of three mirrors. Among them, $M_{3}$ and $M_{4}$ are total reflection mirrors and $M_{2}$ is a mirror with appropriate transmittance. For transmission mirrors, this combination is equivalent to the coupling of two resonant

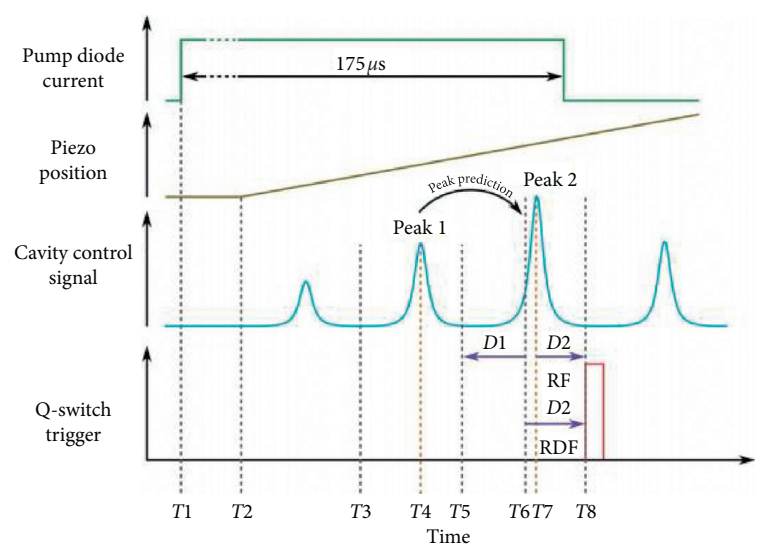

FIGURE 16: Schematic of the trigger mechanism of RDF resonance detection technology [65].

cavities: one resonant cavity is composed of $M_{1}$ and $M_{3}$ with a cavity length of $L_{1}+L_{2}$, and the other resonant cavity is composed of $M_{3}$ and $M_{4}$ with a cavity length of $L_{3}+L_{2}$. If $L_{2}$ and $L_{3}$ are short, a coupling of a short resonant cavity and a long resonant cavity will be formed. The longitudinal mode frequency interval of the short resonant cavity is

$$
\Delta v_{\text {short }}=\frac{c}{2 \mu\left(L_{2}+L_{3}\right)} .
$$

The longitudinal mode frequency interval of the long cavity is

$$
\Delta v_{\text {long }}=\frac{c}{2 \mu\left(L_{1}+L_{2}\right)} \text {. }
$$

where $\mu$ is the refractive index of the gas in the cavity. The light oscillates only when the above two resonance conditions are reached. Therefore, as long as $L_{3}+L_{2}$ is selected to be sufficiently small, a SLM output can be obtained.

The interferometer compound cavity is usually used to generate a SLM laser. The Michelson interference ceremonial compound cavity [67] and the Fox--Smith interference ceremonial compound cavity [68] are two representatives of the interference ceremonial compound cavity. Among allfiber lasers, there are many examples of compound cavities realized by optical fibers, and there are fewer applications of compound cavities in solid-state lasers. In 2016, Bai et al. [69] demonstrated an SLM Q-switched laser based on a three-plan resonant reflector, which is a linear cavity compound cavity. The laser delivers single-pulse energy of $10 \mathrm{~mJ}$ and a pulse width of $10.7 \mathrm{~ns}$ at $10 \mathrm{~Hz}$.

2.7. Nonlinear Frequency Conversion Method. Nonlinear frequency conversions such as frequency doubling, stimulated Raman scattering, and stimulated Brillouin scattering provide a valuable additional mechanism for increasing gain competition and therefore enhancing SLM stability [44, 70, 71]. For example, SLM laser operation can be obtained in simple cavities even without single-mode constraints on the pump laser owing to the SHB free and homogeneous nature of Raman gain. 


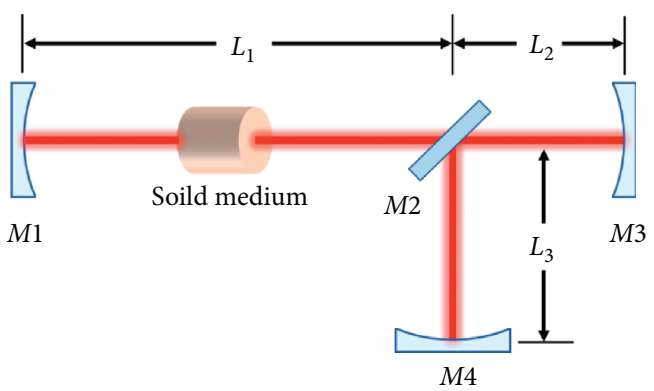

FIGURE 17: Composite cavity structure.

In 2019, Sheng et al. [72] demonstrated a single-frequency intracavity Raman laser based on the spectral cleanup effects in the SHB free SRS gain media. The singlefrequency Raman laser is shown in Figure 18. In their study, multi-watt, stable SLM Stokes $(\sim 1178 \mathrm{~nm})$ was derived from the multimode Nd:GdVO4 fundamental (linewidth $<0.1 \mathrm{~nm}$ @ $1.06 \mu \mathrm{m})$ through hole burning free Raman gain. Then, SLM yellow output at the wavelength of $589.16 \mathrm{~nm}$ is obtained by intracavity frequency-doubling of the Stokes wave when it was tuned to $1178.32 \mathrm{~nm}$.

In 2019, Yang et al. [73] developed a single-frequency diamond Raman laser with output powers of $38 \mathrm{~W}$ at $620 \mathrm{~nm}$ and $11.8 \mathrm{~W}$ at $1240 \mathrm{~nm}$. The Raman cavity is based on a simple standing-wave structure pumped by a multilongitudinal mode laser. Subsequently, the same group [5] demonstrated a SLM $589 \mathrm{~nm}$ laser with near-diffraction limited beam quality generated in a standing-wave diamond Raman resonator with intracavity second-harmonic generation. With a $63 \mathrm{~W} 1018 \mathrm{~nm}$ pump, $589 \mathrm{~nm}$ laser with output power up to $22 \mathrm{~W}$ was achieved. The experimental configuration is shown in Figure 19.

SLM laser operation based on nonlinear frequency conversion can lead to more kinds of frequency lasers. However, this method has a high requirement of nonlinear crystals, and its output mode quality is limited by the material quality and the nonlinear properties of the crystal.

2.8. Multistructure Combination. In practice, due to the processing technology of the parts and the characteristics of the structure itself, a single-mode selection structure is increasingly unable to meet the requirements for SLM selection. To overcome this, multiple structures have been combined to improve the SLM selection level. In 2014, Zhang et al. [74] of the Shanghai Institute of Optics and Mechanics used the torsion cavity structure, combined with the resonance detection technology, to obtain the doublepulse SLM pulse output, as shown in Figure 20. When using dual-crystal RTP electrooptical modulation, the single pulse energy could reach $10 \mathrm{~mJ}$. The pulse width of the generated output was $20 \mathrm{~ns}$, the repetition frequency was $50 \mathrm{~Hz}$, and the optical conversion efficiency and slope efficiency were $22.4 \%$ and $33 \%$, respectively. However, if PZT control the cavity length to meet the resonance conditions, fluctuations will occur, and the hysteresis effect of PZT will reduce the stability. In order to overcome the shortcomings of PZT,
Zhang et al. [75] replaced PZT with electrooptic crystals in 2017. The scanning-delay-triggered resonance detection technology was used to control the cavity length, which improved the stability of the laser. The pulse energy was increased to $13 \mathrm{~mJ}$, the pulse width was $20 \mathrm{~ns}$, the repetition frequency was $50 \mathrm{~Hz}$, and the optical conversion efficiency and slope efficiency were $27 \%$ and $34 \%$, respectively.

In 2019, Hu et al. [76] and others used the RBG and FP etalons to jointly select the mode and used the pressurized Q-switch method. The structure is shown in Figure 21. A stable single frequency could be achieved with a cavity length of $145.7 \mathrm{~mm}$. A pulse of output power of $750 \mathrm{~mW}$, single pulse energy of $75 \mu \mathrm{J}$, frequency $10 \mathrm{kHz}$, and pulse width of $8.3 \mathrm{~ns}$ could be obtained using this technique.

In 2020, Jin et al. [77] proposed a structure in which active and passive dual Q-switching are combined with multiple longitudinal mode selection technologies. This structure is shown in Figure 22. Using a saturable absorption crystal to adjust the cavity loss, combined with a resonant reflector (etalon) and twisted-mode technology, a symmetrical pulse laser output with a single pulse energy of $10.8 \mathrm{~mJ}$, pulse width of $9.8 \mathrm{~ns}$, and an SLM rate of $96.2 \%$ was obtained.

\section{Summary and Outlook}

This review provided an overview of the main SLM selection methods used in lasers, mainly the short cavity method, the etalon method, the traveling wave resonator method, the VBG method, the seed injection method, the compound cavity method, and the nonlinear crystal method. The advantages and disadvantages of different methods are compared in Table 1.

With the advancement of scientific research, driven by the increasing practical demand, the performance of SLM lasers will become progressively better in the future. Miniaturized lasers with high stability, high efficiency, narrow line width, and large energy will become a reality in the future. The seed injection method, nonlinear crystal method, and compound structure method are the frontrunners in future selection technologies. The advantage of the high power of the seed injection method, the nonlinear frequency conversion characteristics of the nonlinear crystal, and the high precision of the composite structure are important factors in practical applications. In addition, 


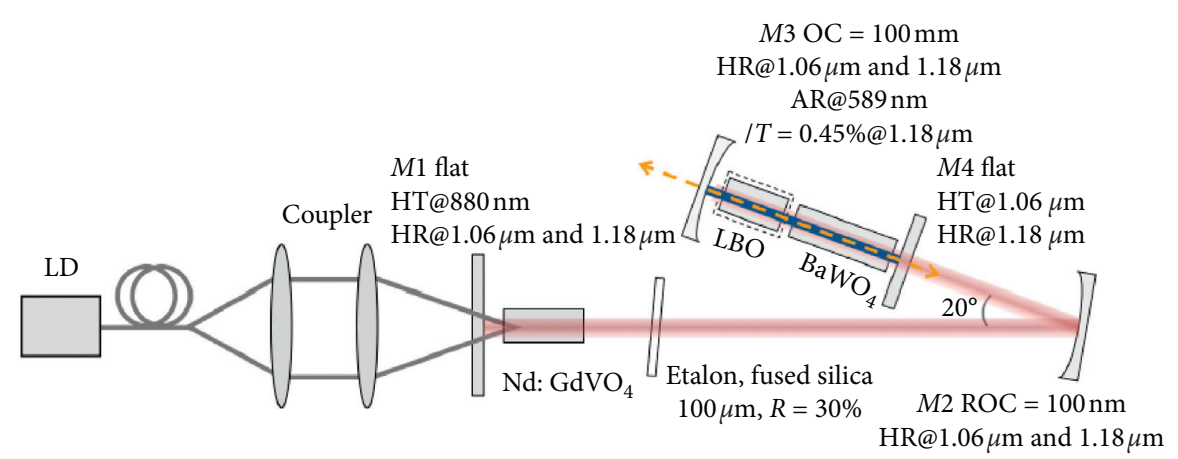

FIGURE 18: Schematic of the single-frequency intracavity Raman laser [72].

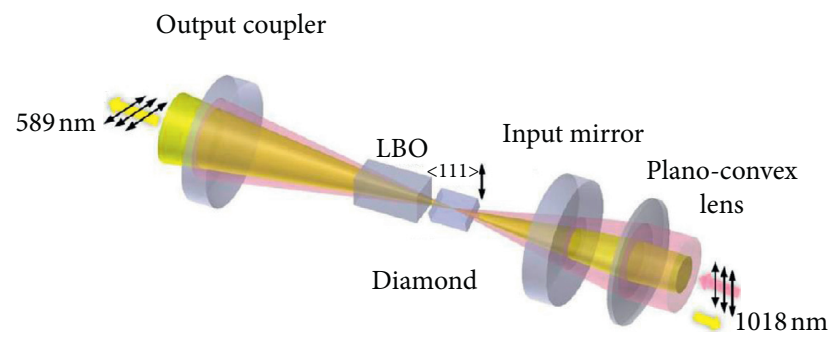

FIGURE 19: Schematic of the $589 \mathrm{~nm}$ diamond Raman laser with intracavity second-harmonic generation [73].

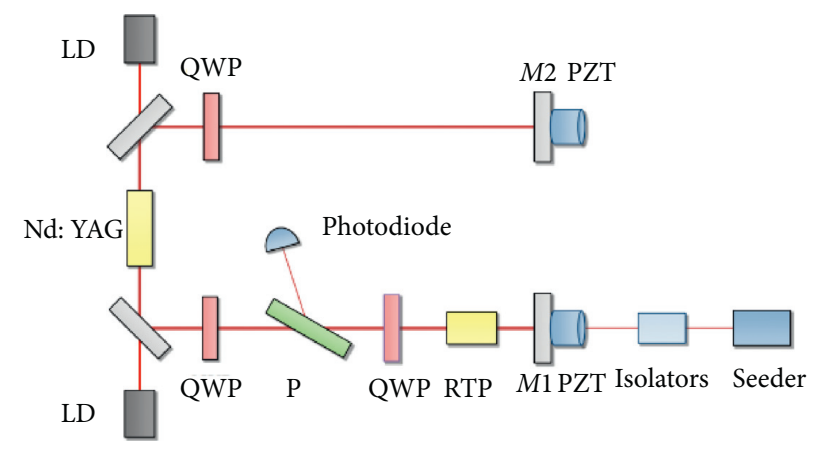

FIGURE 20: Structure of a SLM laser based on torsion mode cavity and resonance detection technology [74].

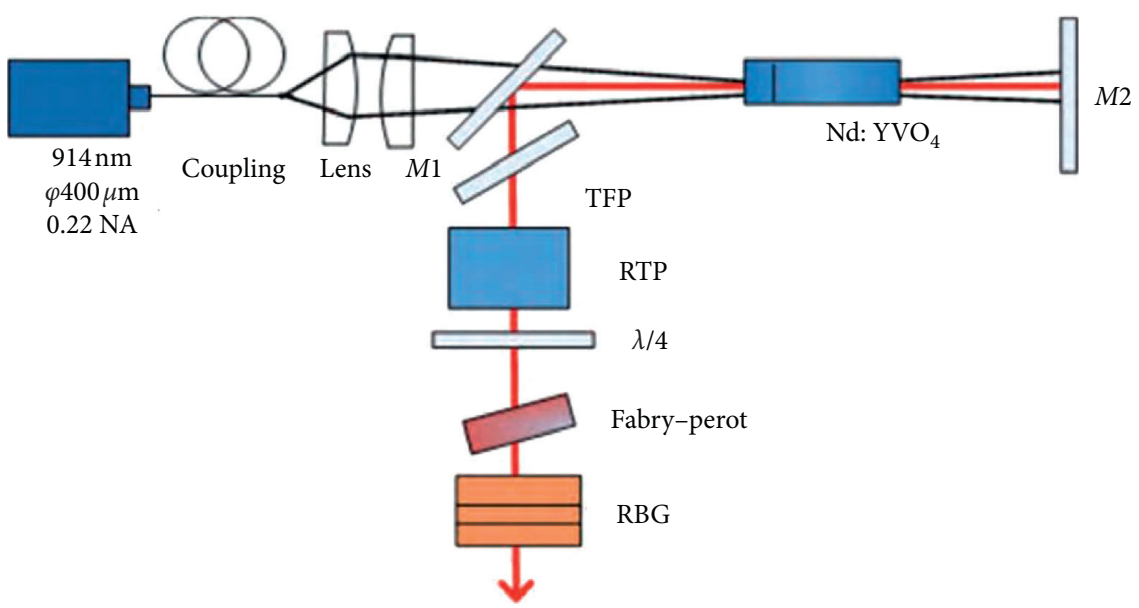

FIGURE 21: Schematic of a SLM laser based on etalon and RBG [77]. 


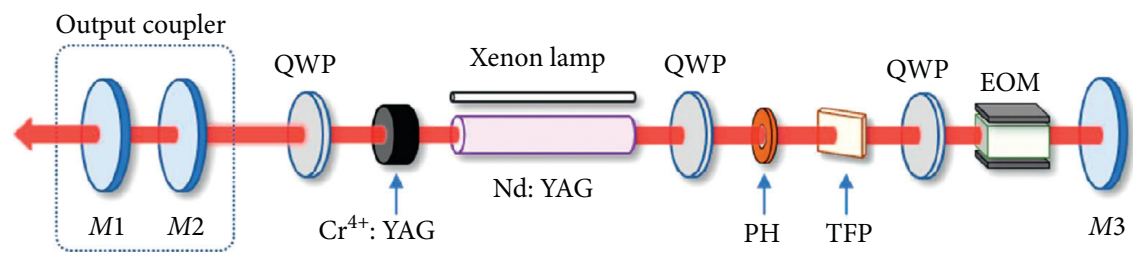

Figure 22: Schematic of dual Q-switched SLM laser [82].

TABLE 1: Comparison of advantages and disadvantages of typical SLM selection technology.

\begin{tabular}{|c|c|c|}
\hline Method & Advantage & Disadvantage \\
\hline Short cavity method & The most direct method, simple structure & Low power, low stability \\
\hline Etalon method & Simple structure can generate relatively high power & Introduce insertion loss, threshold dependent \\
\hline \multicolumn{3}{|l|}{$\begin{array}{l}\text { Traveling wave cavity } \\
\text { method }\end{array}$} \\
\hline Nonplanar & Compact structure, strong stability & High processing requirements, limited tuning range \\
\hline Discrete ring & $\begin{array}{c}\text { A variety of components can be inserted, with good } \\
\text { mode selection effect }\end{array}$ & Complex structure \\
\hline Twisting cavity & Compact structure, easy to realize & $\begin{array}{l}\text { Sensitive to polarization, greatly affected by thermal } \\
\text { effects }\end{array}$ \\
\hline VBG method & Low-temperature influence, high stability & Low precision, limited operating spectral range \\
\hline Seed injection & High-power operation can be achieved & Seed light has a big impact on output \\
\hline $\begin{array}{l}\text { Compound cavity } \\
\text { method }\end{array}$ & Low temperature influence, high stability & Poor selection of single structure \\
\hline Nonlinear crystal method & Frequency conversion & $\begin{array}{c}\text { Relay on the properties of nonlinear optical materials, } \\
\text { quantum defect }\end{array}$ \\
\hline $\begin{array}{l}\text { Multistructure } \\
\text { combination }\end{array}$ & High precision & Complex structure, poor stability \\
\hline
\end{tabular}

the use of fiber resonators in solid-state lasers may also help in making the use of composite resonators more widespread.

\section{Conflicts of Interest}

The authors declare that there are no conflicts of interest regarding the publication of this paper.

\section{Acknowledgments}

This work was supported by the National Key Research \& Development Program of China (2017YFB1104503), KeyArea Research and Development Program of Guangdong Province (2018B090904003), the Natural Science Foundation of Hebei Province (F2019202337), Hebei Foundation for Returned Overseas Scholars (C20190177), Hebei Science and Technology Innovation Strategy Funding Project (20180601), and Key Laboratory of Optoelectronic Information Technology Education (Tianjin University) (2020KFKT005). The authors would like to acknowledge the support from the Innovation and Entrepreneurship Training Program of Hebei University of Technology.

\section{References}

[1] I. A. Razenkov, "Aerosol lidar for continuous atmospheric monitoring," Atmospheric and Oceanic Optics, vol. 26, no. 4, pp. 308-319, 2013.

[2] M. Frede, R. Wilhelm, R. Gau et al., "High-power singlefrequency Nd: YAG laser for gravitational wave detection,"
Classical and Quantum Gravity, vol. 21, no. 5, pp. S895-S901, 2004.

[3] Y. H. Lu, L. Zhang, X. F. Xu et al., "208 W all-solid-state sodium guide star laser operated at modulated-longitudinal mode," Optics Express, vol. 27, no. 15, Article ID 20282, 2019.

[4] J. Zhou and X. Long, "Laser Doppler velocimeter using a single longitudinal mode solid-state laser source," Optics \& Laser Technology, vol. 42, no. 7, pp. 1167-1171, 2010.

[5] X. Yang, O. Kitzler, D. J. Spence, Z. Bai, Y. Feng, and R. P. Mildren, "Diamond sodium guide star laser," Optics Letters, vol. 45, no. 7, pp. 1898-1901, 2020.

[6] N. Vorobiev, L. Glebov, and V. Smirnov, "Single-frequency-mode Q-switched Nd: YAG and Er: glass lasers controlled by volume Bragg gratings," Optics Express, vol. 16, no. 12, pp. 9199-9204, 2008.

[7] C. Cui, Y. Wang, Z. Lu et al., "High-visibility pseudothermal light source based on a $\mathrm{Cr}^{4+}$ : YAG passively Q-switched single-longitudinal-mode laser," International Journal of Optics, vol. 2020, Article ID 3160837, 2020.

[8] Z. Bai, H. Chen, X. Gao, S. Li, Y. Qi, and Z. Bai, "Highly compact nanosecond laser for space debris tracking," Optical Materials, vol. 98, p. 109470, 2019.

[9] S. Li, Y. Wang, Z. Lu et al., "Hundred-Joule-level, nanosecond-pulse Nd: glass laser system with high spatiotemporal beam quality," High Power Laser Science and Engineering, vol. 4, Article ID e10, 2016.

[10] P. Loiko, R. Soulard, G. Brasse et al., "Passive Q-switching of a $\mathrm{Tm}^{3+}: \mathrm{LiYF}_{4}$ waveguide laser by $\mathrm{Cr}^{2+}: \mathrm{ZnSe}$ and $\mathrm{Co}^{2+}: \mathrm{ZnSe}$ saturable absorbers," Optical Materials, vol. 107, Article ID 110116, 2020.

[11] R. Paschotta, Field Guide to Lasers, SPIE Press, Bellingham, WA, USA, 2008. 
[12] B. Q. Yao, X. L. Liu, L. X. Yu et al., "Resonantly pumped single frequency Er: YAG laser at $1645 \mathrm{~nm}$," Laser Physics, vol. 22, no. 2, pp. 403-405, 2011.

[13] A. J. Merriam and G. Y. Yin, "Efficient self-seeding of a pulsed $\mathrm{Ti}^{3+}: \mathrm{Al}_{2} \mathrm{O}_{3}$ laser," Optics Letters, vol. 23, no. 13, pp. 1034-1036, 1998.

[14] Y. F. Chen, T. M. Huang, C. L. Wang, L. J. Lee, and S. C. Wang, "Theoretical and experimental studies of singlemode operation in diode pumped Nd: YVO4/KTP green laser: influence of KTP length," Optics Communications, vol. 152, no. 4-6, pp. 319-323, 1998.

[15] F. Zhou and A. I. Ferguson, "Tunable single frequency operation of a diode laser pumped Nd:YAG microchip at $1.3 \mu \mathrm{m}$," Electronics Letters, vol. 26, no. 7, pp. 490-491, 1990.

[16] J. J. Zayhowski and A. Mooradian, "Single-frequency microchip Nd lasers," Optics Letters, vol. 14, no. 1, pp. 24-26, 1989.

[17] T. J. Kane and R. L. Byer, "Monolithic, unidirectional singlemode Nd: YAG ring laser," Optics Letters, vol. 10, no. 2, pp. 65-67, 1985.

[18] V. Byer and A. E. Siegman, "A "twisted-mode" technique for obtaining axially uniform energy density in a laser cavity," Applied Optics, vol. 4, no. 1, pp. 142-143, 1965.

[19] K. Kubodera and K. Otsuka, "Efficient $\mathrm{LiNdP}_{4} \mathrm{O}_{12}$ lasers pumped with a laser diode," Applied Optics, vol. 18, no. 23, pp. 3882-3883, 1979.

[20] G. Li, B. Q. Yao, C. H. Zhang et al., "Diode pumped operation of Tm, Ho: $\mathrm{YVO}_{4}$ microchip laser," Chinese Physics Letters, vol. 27, no. 3, pp. 152-154, 2010.

[21] Z. G. Wang, B. Q. Yao, G. Li, Y. L. Ju, and Y. Z. Wang, "Single longitudinal mode lasing of Tm, Ho: YAP microchip laser at 2000.4 nm," Laser Physics, vol. 20, no. 2, pp. 458-461, 2010.

[22] Z. You, Y. Wang, J. Xu et al., "Single-longitudinal-mode Er: GGG microchip laser operating at $27 \mu \mathrm{m}$," Optics Letters, vol. 40, no. 16, pp. 3846-3849, 2015.

[23] Y. Chen, Y. Lin, J. Huang, X. Gong, Z. Luo, and Y. Huang, "Single-longitudinal-mode $1521 \mathrm{~nm}$ passively $q$-switched Er: Yb: YAl3 (BO3)4 pulse microchip laser," Optics Express, vol. 27, no. 18, pp. 26080-26086, 2019.

[24] N. Li, W. M. Wang, Y. H. Lu et al., "Tunable linewidth control technique for solid-state laser based on Fabry-Perot etalon," High Power Laser and Particle Beams, vol. 25, no. 5, pp. 1139-1143, 2013.

[25] Y. Y. Wang, J. H. Liu, S. C. Li et al., "Stable and simple structure passively $Q$-switched single-longitudinal-mode laser," Chinese Journal of Lasers, vol. 31, no. 5, pp. 531-534, 2004.

[26] M. Born and E. Wolf, Principles of Optics, PERGAMON, Bergama, Turkey, 1959.

[27] S. A. Collins and G. R. White, "Interferometer laser mode selector," Applied Optics, vol. 2, no. 4, pp. 448_1-449, 1963.

[28] H. Danielmeyer, "Stabilized efficient single-frequency $\mathrm{Nd}$ : YAG laser," IEEE Journal of Quantum Electronics, vol. 6, no. 2, pp. 101-104, 1970.

[29] B. Q. Yao, L. Ke, X. M. Duan et al., "Stable wavelength narrow linewidth diode-pumped Tm: YLF laser with double etalons," Laser Physics Letters, vol. 6, no. 8, pp. 563-566, 2010.

[30] X. L. Zhang, L. Li, J. H. Cui, Y. L. Ju, and Y. Z. Wang, "Single longitudinal mode and continuously tunable frequency Tm, Ho: YLF laser with two solid etalons," Laser Physics Letters, vol. 7, no. 3, pp. 194-197, 2010.

[31] Y. Deng, B. Q. Yao, Y. L. Ju et al., “A diode-pumped $1617 \mathrm{~nm}$ single longitudinal mode Er: YAG laser with intra-cavity etalons," Chinese Physics Letters, vol. 31, no. 7, Article ID 074202, 2014.

[32] W. Liu, Y. L. Ju, T. Y. Dai et al., "Single-longitudinal-mode Ho: LuAG laser at $2.1 \mu \mathrm{m}$," Laser Physics, vol. 26, no. 2, Article ID 025002, 2016.

[33] A. C. Nilsson, E. K. Gustafson, and R. L. Byer, "Eigenpolarization theory of monolithic nonplanar ring oscillators," IEEE Journal of Quantum Electronics, vol. 25, no. 4, pp. 767-790, 1989.

[34] T. J. Kane, A. C. Nilsson, and R. L. Byer, "Frequency stability and offset locking of a laser-diode-pumped Nd: YAG monolithic nonplanar ring oscillator," Optics Letters, vol. 12, no. 3, pp. 175-177, 1987.

[35] L. Wang, C. Gao, M. Gao, and Y. Li, "Resonantly pumped monolithic nonplanar Ho:YAG ring laser with high-power single-frequency laser output at $2122 \mathrm{~nm}$," Optics Express, vol. 21, no. 8, pp. 9541-9546, 2013.

[36] C. Gao, M. Gao, Y. Zhang, Z. Lin, and L. Zhu, "Stable singlefrequency output at $201 \mu \mathrm{m}$ from a diode-pumped monolithic double diffusion-bonded Tm: YAG nonplanar ring oscillator at room temperature," Optics Letters, vol. 34, no. 19, pp. 3029-3031, 2009.

[37] R. Wang, C. Gao, Y. Zheng, M. Gao, and Q. Ye, “A resonantly pumped $1645 \mathrm{~nm}$ Er: YAG nonplanar ring oscillator with $10.5 \mathrm{~W}$ single frequency output," IEEE Photonics Technology Letters, vol. 25, no. 10, pp. 955-957, 2013.

[38] M. W. Gao, Y. Zhao, L. Y. Zhang et al., " $1319 \mathrm{~nm}$ singlefrequency output from diffusion-bonded monolithic nonplanar Nd: YAG ring resonator with undoped end," Chinese Physics Letters, vol. 11, no. 4, Article ID 041406, 2013.

[39] W. Deng, T. Yang, J. Cao et al., "High-efficiency $1064 \mathrm{~nm}$ nonplanar ring oscillator Nd: YAG laser with diode pumping at $885 \mathrm{~nm}$," Optics Letters, vol. 43, no. 7, pp. 1562-1565, 2018.

[40] A. R. Clobes and M. J. Brienza, "Single-frequency travelingwave Nd: YAG laser," Applied Physics Letters, vol. 21, no. 6, pp. 265-267, 1972.

[41] J. Q. Zhao, Y. Z. Wang, B. Q. Yao, and Y. L. Ju, "High efficiency, single-frequency continuous wave Nd: YVO4/ YVO4ring laser," Laser Physics Letters, vol. 7, no. 2, pp. 135-138, 2010.

[42] P. C. Shardlow and M. J. Damzen, "High efficiency $17 \mathrm{~W}$ single frequency ring laser with feedback mirror," in Proceedings of the European Conference on Lasers and ElectorOptics 2009 and European Quantum Electronics Conference, Munich, Germany, December 2009.

[43] W. F. Zhao, W. Hou, L. Guo et al., " 12 W high efficiency single frequency ring laser," Laser Physics Letters, vol. 7, no. 3, pp. 201-212, 2010.

[44] K. I. Martin, W. A. Clarkson, and D. C. Hanna, "Self-suppression of axial mode hopping by intracavity second-harmonic generation," Optics Letters, vol. 22, no. 6, pp. 375-377, 1997.

[45] J. Liu, Z. Wang, H. Li, Q. Liu, and K. Zhang, "Stable, 12 W, continuous-wave single-frequency Nd: YVO_4 green laser polarized and dual-end pumped at $880 \mathrm{~nm}$," Optics Express, vol. 19, no. 7, pp. 6777-6782, 2011.

[46] Y. Wang, W. Yang, H. Zhou, M. Huo, and Y. Zhen, "Temperature dependence of the fractional thermal load of $\mathrm{Nd}$ : YVO_4 at $1064 \mathrm{~nm}$ lasing and its influence on laser performance," Optics Express, vol. 21, no. 15, pp. 18068-18078, 2013.

[47] E. Wu, H. Pan, S. Zhang, and H. Zeng, "High power singlelongitudinal-mode operation in a twisted-mode-cavity laser with a c-cut Nd: $\mathrm{GdVO}_{4}$ crystal," Applied Physics B, vol. 80, no. 4-5, pp. 459-462, 2005. 
[48] C. Gao, R. Wang, Z. Lin et al., "2 $\mu \mathrm{m}$ single-frequency Tm: YAG laser generated from a diode-pumped $L$-shaped twisted mode cavity," Applied Physics B, vol. 107, no. 1, pp. 67-70, 2012.

[49] S. Luo, Z. Cai, H. Xu et al., "Direct oscillation at $640 \mathrm{~nm}$ in single longitudinal mode with a diode-pumped Pr: YLF solidstate laser," Optics \& Laser Technology, vol. 116, pp. 112-116, 2019.

[50] Z. Sun, Q. Li, H. Lei, Y. Hui, and M. Jiang, "Sub-nanosecond pulse, single longitudinal mode Q-switched Nd: YVO4 laser controlled by reflecting Bragg gratings," Optics \& Laser Technology, vol. 48, pp. 475-479, 2013.

[51] C. J. Jin, D. Li, Y. Bai et al., "Wideband tunable graphenebased passively Q-switched Tm: YAP laser," Laser Physics, vol. 25, no. 4, Article ID 045802, 2015.

[52] Y. L. Hui, Q. Li, X. Zhang et al., "Single frequency mode controlled by volume bragg gratings," Chinese Journal of Lasers, vol. 36, no. 11, pp. 2805-2807, 2009.

[53] Y. Park, G. Giuliani, and R. Byer, "Single axial mode operation of a Q-switched Nd: YAG oscillator by injection seeding," IEEE Journal of Quantum Electronics, vol. 20, no. 2, pp. 117-125, 1984.

[54] T. Schröder, C. Lemmerz, O. Reitebuch, M. Wirth, C. Wührer, and R. Treichel, "Frequency jitter and spectral width of an injection-seeded Q-switched Nd: YAG laser for a Doppler wind lidar," Applied Physics B, vol. 87, no. 3, pp. 437-444, 2007.

[55] S. W. Henderson, E. H. Yuen, and E. S. Fry, "Fast resonancedetection technique for single-frequency operation of injection-seeded Nd: YAG lasers," Optics Letters, vol. 11, no. 11, pp. 715-717, 1986.

[56] Q. Na, C. Gao, Q. Wang et al., "1 kHz single-frequency $209 \mu \mathrm{m}$ Ho: YAG ring laser," Applied Optics, vol. 56, no. 25, pp. 7075-7078, 2017.

[57] E. S. Fry, E. Thomas, M. P. Larsen et al., "Injection seeding of a Ti: sapphire laser using a ramp-hold-fire technique," in Proceedings of the Lasers and Elector-Optics, pp. 362-363, Baltimore, MD, USA, May 1997.

[58] T. Walther, M. P. Larsen, and E. S. Fry, "Generation of Fourier-transform-limited 35-ns pulses with a ramp-hold-fire seeding technique in a Ti:sapphire laser," Applied Optics, vol. 40, no. 18, pp. 3046-3050, 2001.

[59] Y. X. Zhang, C. Q. Gao, S. Wang et al., "Single-frequency, injection-seeded $Q$-switched Ho: YAG ceramic laser pumped by a $1.91 \mu \mathrm{m}$ fiber-coupled LD," Optics Express, vol. 122, no. 7, p. 198, 2016.

[60] K. Ertel, H. Bösenberg, and J. Bosenberg, "Injection-seeded pulsed Ti:sapphire laser with novel stabilization scheme and capability of dual-wavelength operation," Applied Optics, vol. 44, no. 24, pp. 5120-5126, 2005.

[61] J. Zhou, T. Yu, J. Q. Liu et al., "Development of single-frequency laser for direct-detection wind lidar," Proceedings of SPIE, vol. 44, no. 24, pp. 5120-5126, 2007.

[62] J. Zhou, H. G. Zang, D. Liu et al., "Frequency doubled singlelongitudinal-mode Nd: YAG laser for remote sensing," in Proceedings of the 24th International Laser Radar Conference, pp. 133-136, Boulder, CO, USA, June 2008.

[63] Y. F. Gao, J. X. Zhang, H. G. Zang et al., "Stable single-mode operation of injection-seeded Q-switched Nd: YAG laser by sine voltage modulation," Chinese Optics Letters, vol. 14, no. 7, Article ID 071401, 2016.

[64] F. Gibert, D. Edouart, C. Cénac, and F. Le Mounier, " $2 \mu \mathrm{m}$ high-power multiple-frequency single-mode Q-switched Ho:
YLF laser for DIAL application," Applied Physics B, vol. 116, no. 4, pp. 967-976, 2014.

[65] C. Lemmerz, O. Lux, O. Reitebuch, B. Witschas, and C. Wührer, "Frequency and timing stability of an airborne injection-seeded Nd: YAG laser system for direct-detection wind lidar," Applied Optics, vol. 56, no. 32, pp. 9057-9068, 2017.

[66] J. X. Long, G. Li, B. Yang et al., "Progress in injection-seeded all-solid-state single-frequency pulse laser," Laser \& Optoelectronics Progress, vol. 55, no. 09, pp. 7-14, 2018.

[67] Y. D. Kolomnikov, V. N. Lisits, and V. P. Chebotayev, "Laser michelsoninterferometer," Optics \& Spectroscopy, vol. 22, 1967.

[68] P. Smith, "Stabilized single-frequency output from a long ring laser," IEEE Journal of Quantum Electronics, vol. 4, no. 8, pp. 485-490, 1968.

[69] Z. Bai, Y. Wang, Z. Lu et al., “A single-longitudinal-mode Nd: Ce: YAG Q-switched laser based on a three-plan resonant reflector," Journal of Russian Laser Research, vol. 37, no. 4, pp. 382-388, 2016.

[70] S. Greenstein and M. Rosenbluh, "The influence of nonlinear spectral bandwidth on single longitudinal mode intra-cavity second harmonic generation," Optics Communications, vol. 248, no. 1-3, pp. 241-248, 2005.

[71] Z. Bai, H. Yuan, Z. Liu et al., "Stimulated brillouin scattering materials, experimental design and applications: a review," Optical Materials, vol. 75, pp. 626-645, 2018.

[72] Q. Sheng, H. Ma, R. Li et al., "Recent progress on narrowlinewidth crystalline bulk Raman lasers," Results in Physics, vol. 17, Article ID 103073, 2020.

[73] X. Yang, O. Kitzler, D. J. Spence et al., "Single-frequency $620 \mathrm{~nm}$ diamond laser at high power, stabilized via harmonic self-suppression and spatial-hole-burning-free gain," Optics Letters, vol. 44, no. 4, pp. 839-842, 2019.

[74] J. X. Zhang, S. G. Li, X. L. Zhu et al., "Single-frequency doublepulse Q-switched Nd: YAG laser with a RTP electro-optic modulater," Advanced Solid State Lasers, vol. 20, 2014.

[75] J. Zhang, X. Zhu, H. Zang et al., "Double-pulse singlelongitudinal-mode operation of injection-seeded laser using intracavity phase modulator," Optical Engineering, vol. 56, no. 4, Article ID 046105, 2017.

[76] X. Hu, D. J. Cheng, S. B. Wang et al., "Single-frequency Nd: $\mathrm{YVO}_{4}$ laser based on reflective Bragg grating combined with fabry-perot etalon," Acta Optica Sinica, vol. 39, no. 05, pp. 228-233, 2019.

[77] D. Jin, Z. X. Bai, Q. Z. Wang et al., "Doubly Q-switched single longitudinal mode $\mathrm{Nd}$ : YAG laser with electro-optical modulator and $\mathrm{Cr}^{4+}$ : YAG," Optics Communications, vol. 463, Article ID 125500, 2020.

[78] T. T. Lu, J. T. Wang, X. L. Zhu et al., "Highly efficient single longitudinal mode-pulsed green laser," Chinese Optics Letters, vol. 11, no. 5, Article ID 051402, 2013.

[79] I. G. Marienko, M. Zhi, A. I. Khizhnyak, and A. V. Sokolov, "Injection-seeded single-longitudinal-mode Ti: sapphire laser with no active stabilization," Optics Express, vol. 28, no. 17, pp. 25444-25459, 2020. 